\title{
Film Growth Mechanisms in Pulsed Laser Deposition
}

\section{Citation}

Aziz, Michael J. 2008. Film growth mechanisms in pulsed laser deposition. Applied Physics A:

Materials Science \& Processing 93(3): 579-587.

\section{Published Version}

http://dx.doi.org/10.1007/s00339-008-4696-7

\section{Permanent link}

http://nrs.harvard.edu/urn-3:HUL.InstRepos:2795447

\section{Terms of Use}

This article was downloaded from Harvard University's DASH repository, and is made available under the terms and conditions applicable to Open Access Policy Articles, as set forth at http:// nrs.harvard.edu/urn-3:HUL.InstRepos:dash.current.terms-of-use\#OAP

\section{Share Your Story}

The Harvard community has made this article openly available.

Please share how this access benefits you. Submit a story.

\section{Accessibility}


Applied Physics A, in press (2008), Submitted October 13, 2007

\section{Film Growth Mechanisms in Pulsed Laser Deposition}

Michael J. Aziz, Harvard School of Engineering and Applied Sciences, Cambridge MA 02138 USA

\section{ABSTRACT}

This paper reviews our recent studies of the fundamentals of growth morphology evolution in Pulsed Laser Deposition in two prototypical growth modes: metal-on-insulator island growth and semiconductor homoepitaxy. By comparing morphology evolution for pulsed laser deposition and thermal deposition in the same dual-use chamber under identical thermal, background, and surface preparation conditions, and varying the kinetic energy by varying the laser fluence or using an inert background gas, we have isolated the effect of kinetic energy from that of flux pulsing in determining the differences between morphology evolution in these growth methods. In each growth mode analytical growth models and Kinetic Monte Carlo simulations for thermal deposition, modified to include kinetic energy effects, are successful at explaining much of what we observe experimentally.

\section{INTRODUCTION}

For the fabrication of new materials and assemblies of materials, we often find ourselves saying "If I could only make this particular structure, I bet it would have these wonderful properties." But how can we place and hold the atoms where we want them to be? The revolution 
in materials processing that has occurred over the past quarter century has ushered in a host of new processing techniques, many of which accomplish just this because, by design or accident, they control the kinetics to produce materials that are permanently stuck out of thermodynamic equilibrium. Our understanding of the kinetic laws dictating the final product that forms under any particular processing conditions has, in general, lagged far behind the empiricism that has guided the development and use of processing techniques. This situation is to be contrasted with the current status of synthetic organic chemistry: because organic chemical reaction mechanisms and kinetics are so well understood, chemists are able to synthesize, deliberately and rationally, an almost unlimited variety of organic structures. Empiricism can only get you so far before diminishing returns make things difficult. Sooner or later a fundamental understanding of the phenomenology and mechanisms involved is needed. This permits the intelligent generalization of the process to untested length scales, to untested materials, and to related processes, greatly enhancing our capabilities for continued progress. The alternative is the empirical exploration of a vast parameter space experimentally - sometimes at great temporal and financial cost.

In this paper I review our recent studies of the fundamentals of growth morphology evolution in Pulsed Laser Deposition (PLD), with an emphasis on a comparison with Molecular Beam Epitaxy (MBE) or Physical Vapor Deposition (PVD). MBE / PVD is an ideal foundation upon which to build because there is now a solid baseline of knowledge about surface structures; stress effects; atomistic mechanisms; growth modes; and the incorporation of dopants, impurities, and alloying elements during growth. Two essential differences between PLD and MBE are widely recognized: (1) in PLD the depositing species arrive in short bursts, on the order of 10-100 $\mu$ s, instead of in steady state; and (2) in PLD the depositing species have kinetic energy of order 10$100 \mathrm{eV}$ — some two orders of magnitude greater than in MBE. One of our goals has been to 
determine the relative contributions of these features in determining the distinctive aspects of PLD growth morphologies.

PLD uses a pulsed laser to ablate a target to produce the depositing flux[1,2], and has several distinct characteristics advantageous for the understanding of non-equilibrium growth from the vapor. One particularly dramatic difference between crystal growth in MBE and in PLD is the instantaneous deposition rate. In MBE a typical growth rate might be only 1 monolayer (ML) per second, and near-equilibrium growth often occurs[3]. In PLD, one can grow films at these average rates, but commonly the instantaneous rate is some 3-5 orders of magnitude faster. The average growth speed is limited only by the repetition rate of the laser, which can be changed abruptly without significant time lags. Hence, PLD growth is an area of opportunity for a variety of fundamental kinetic studies that are difficult in MBE growth. The characteristics that make it particularly interesting are:

1. PLD consists of periodic bursts of highly driven growth followed by relatively long periods of uninterrupted surface relaxation, permitting these two competing processes to be isolated and studied separately.

2. In the proper ablation regime, ionized and neutral ablation products having kinetic energies in the range from less than one to a few hundred eV can be produced[4-6]. The variable kinetic energy can be used to study a variety of phenomena such as enhanced lowtemperature epitaxy and surface segregation/incorporation reactions.

3. The instantaneous deposition flux can be varied independently of either the kinetic energy of the ablated species, the average growth rate, or the average atomic mobility on the surface. 
Additionally, there are a number of practical advantages of PLD, including "congruent transfer" from the target (under some circumstances [7]); layer-by-layer control by using multiple targets sequentially; the ability to ablate virtually any target; and the ability to deposit in reactive atmospheres for doping, alloying, or compound formation.

The development of PLD technology began at about the same time as that of MBE. However, although MBE has moved into production facilities, the PLD process remained largely in the laboratory. The reasons for this are tied to the historical development of PLD[8]. The deposition of inorganic materials by PLD began to be studied shortly after the development of the pulsed ruby laser, and, by 1970, a full complement of III-V and II-VI semiconductor thin films had been grown. Although these films were uniform and had the same composition as the target material, they were grown on glass and quartz substrates, thus were polycrystalline and not suited for semiconductor devices. During the same time period, the use of $\mathrm{CW}$ lasers (e.g., $\mathrm{CO}_{2}$ ) as a heating source was also investigated, but it was found that evaporation of multicomponent targets using the CW laser was not congruent. The flexibility of the PLD technique was demonstrated as early as 1976 when multiple targets were used to grow superlattices; however, once again the choice of substrates was less than ideal, and polycrystalline and amorphous films resulted. During the early 1980’s the development of pulsed UV excimer lasers had progressed to the point that short pulsed (few tens of ns), high power (tens of MW) lasers became available commercially. During the mid 1980’s these lasers were "married" with the deposition technologies developed for MBE and CVD growth, with the result being that PLD emerged as an alternative deposition process. PLD became popular when it was very successfully used to grow thin, stoichiometric, epitaxial films of the high temperature $\left(\mathrm{HT}_{\mathrm{c}}\right)$ superconducting oxides. 
Today, PLD is used widely for the deposition of $\mathrm{HT}_{\mathrm{c}}$ films, as well as for the growth of dielectrics, ferroelectrics, and other materials with complex composition[9].

There has been much valuable PLD research centered on questions such as "what new material can be grown?" and "what processing conditions optimize the properties of the grown film?". The primary focus of such research clearly has been centered on the development of the PLD technology (e.g., elimination of particulates in deposition[10,11]), to the extent that research on the fundamental issues of film growth in PLD has remained relatively underdeveloped, especially experimentally. For example, consider item \#1 above. Simulation has been an important tool to isolate the effects of deposition and relaxation[12,13], but experimental research involving modulating the deposition rate or temperature in MBE[14-20] is difficult. PLD readily permits us to study these two processes independently.

We have been studying two prototypical growth modes: metal-on-insulator island growth and semiconductor homoepitaxy. The particular emphasis has been the development of a fundamental understanding of the phenomenology and mechanisms underlying growth morphology evolution. Because we emphasize the phenomena, the most effective progress is made in well-studied elemental materials with simple crystal structures — such as Ag and Ge for which the "baseline" for new phenomena is well understood, including the morphology evolution in thermal deposition and the values of important materials parameters. For both growth modes, our efforts have been aimed at answering the question, "to what extent can the 'MBE paradigm' of adatoms, islands, steps, and terraces be utilized to account for the growth morphologies observed in PLD? For each growth mode, by comparing morphology evolution for PLD and thermal deposition in the same dual-use chamber under identical thermal, background, and surface preparation conditions, we have isolated the effect of kinetic energy from that of flux 
pulsing in determining the differences between morphology evolution in PLD and MBE/PVD.

We have then been able to adapt thermal deposition models based on the MBE paradigm by adding kinetic energy effects.

\section{SEMICONDUCTOR HOMOEPITAXY}

There have been reports of improved epitaxial growth characteristics[21] in PLD compared to MBE. Defect reduction for Si has been attributed to improved layer-by-layer growth in PLD[22]. Fe and Co on Cu exhibit improved magnetic properties[23-28] when deposited by PLD. In the case of Fe on $\mathrm{Cu}(111)$, heteroepitaxial growth by PLD has been directly observed to result in improved layer-by-layer growth over thermal deposition [23]. These studies were limited to the first few monolayers of deposition and focused primarily on magnetic properties rather than on growth mechanisms. Kinetic Monte Carlo (KMC) simulations of pulsed flux [29] and pulsed flux with candidate energetic mechanisms [30] indicate that — under the conditions examined — pulsing a thermal flux is likely to lead to increased roughening, and energetic mechanisms are necessary to obtain enhanced smoothening except under conditions unlikely to be attainable experimentally.

As shown in Fig. 1, in semiconductor homoepitaxy for both PLD and MBE the morphology evolution is characterized by increasing surface roughness as growth mounds develop with a well-characterized lateral separation, followed by the evolution of a pyramidal mound morphology, followed by epitaxial breakdown and the formation of an amorphous phase[31]. In Ge homoepitaxy we found that for PLD kinetic energies up to about $300 \mathrm{eV}$, the morphology in PLD and MBE goes through the same qualitative stages [32]. We quantitatively compared 
growth morphology and epitaxial breakdown in (a) PLD with peak kinetic energy 300 eV (PLD-KE); (b) PLD with suppressed kinetic energy comparable to the thermal evaporation energy (PLD-TH); and (c) MBE. As is shown in Fig. 2, the thicknesses at which epitaxial breakdown occurs are ranked in the order PLD-KE > MBE > PLD-TH; also, the surface is smoother in PLD-KE than in MBE [31]. In fact, we found no limit to the epitaxial thickness in PLD-KE. We found that the early occurrence of epitaxial breakdown in PLD-TH is consistent with the kinetics of MBE in pulses but with an instantaneous deposition rate accelerated by a factor of 500, and separated by an inter-pulse period of negligible relaxation. These and other results demonstrate that the enhancement of epitaxial growth - the reduction in roughness and the delay of epitaxial breakdown - are due to the high kinetic energy of depositing species in PLD.

We used quantitative Reflection High Energy Electron Diffraction intensity oscillations to study the first few monolayers of deposition, delineated the necessary conditions for avoiding interference from Kikuchi features [33], and developed a new diffraction model for the intensity during multi-monolayer deposition [34] that permitted us to determine the height of the EhrlichSchwoebel step-edge attachment barrier which causes the growth instability leading to mounds in the topography [35].

The homoepitaxial breakdown mechanism of Ge (001) MBE [36] is that roughening during growth leads to sufficiently high slopes that eventually $\{111\}$ stacking faults readily form, and their accumulation leads eventually to growth of an amorphous phase. We can explain what we observe in our comparison of PLD and MBE by starting with this mechanism and adding energetic mechanisms in the spirit of those invoked to explain enhanced smoothening in sputter deposition [37]: the kinetic energy in the depositing species facilitates the filling of the gap 
between the growth mounds. This occurs possibly by downhill momentum transfer [37], possibly by creating more mobile surface species, and possibly by small island breakup [30] or transient enhanced mobility $[30,38]$ — we have not been able to distinguish between these. More efficient gap filling delays the time at which the growth surface becomes sufficiently misoriented that $\{111\}$ stacking faults can form, thereby delaying epitaxial breakdown.

\section{METAL-ON-INSULATOR ISLAND GROWTH}

The Volmer-Weber growth mode of isolated 3D islands on an otherwise bare substrate is common in the deposition of dissimilar materials [39]. As shown in Fig. 3, in metal-on-insulator film growth the morphology evolution is characterized by a transition from isolated, equiaxed islands to elongated islands to multiply-connected non-percolating islands to a percolating metal film to the filling in of holes. Although the delay by kinetic processes of the morphology evolution toward a uniform, pinhole-free film has often been viewed as a nuisance, recent discoveries of surface plasmon-enhanced phenomena present opportunities for the exploitation of nanoparticulate and nanoporous metal films [40,41].

The morphological progression is consistent with the following picture. As isolated islands grow larger with further deposition, they impinge upon each other and begin to coalesce, driven by capillary forces toward a more equiaxed equilibrium shape — a process that delays the development of a contiguous film. The kinetics of this process have been addressed for continuous deposition [42-44]. The time required for coalescence increases with increasing island size, varying as the fourth power of island radius for surface diffusion mediated coalescence driven by classical capillarity [45]. For a given cluster of two or more coalescing 
islands, there is an island size above which the time required for coalescence exceeds the average time interval before an additional island impinges with one of the constituent islands in the cluster. It is beyond this point that clusters of coalescing islands remain elongated on the surface: they have undergone a kinetic freezing transition [42]. Further deposition joins these elongated clusters, forming a tortuous network of island chains that eventually conducts electrically (the "percolation transition"). With further deposition, the intervening bare channels continue to fill in until no pinholes remain [46].

Pulsing of the deposition flux to manipulate island nucleation and growth has been investigated theoretically by Jensen and coworkers $[47,48]$, who focused on the island size distribution prior to significant impingement, but nevertheless identified three broadly applicable growth regimes when the lifetime of an adatom on the substrate surface is: much shorter than the pulse duration; in between the pulse duration and the pulse period; and much longer than the pulse period. They found different scaling behavior for the island density vs. pulse frequency in these three regimes. We find some of the same scaling behavior in our experiments, despite the importance of impingement and coalescence in our experimental morphologies [49].

Experimentally, island and film morphologies were observed by ex-situ Atomic Force Microscopy (AFM) after growth and quenching to room temperature; additionally the percolation transition was monitored in situ by time-resolved lateral electrical conductance measurements $[49,50]$.

We developed KMC simulations of island nucleation, growth, impingement and coalescence during flux pulsing that neglected any effect of kinetic energy [49]. The rules were:

1. Irreversible adatom aggregation into islands, which are constrained to be hemispherical;

2. Coalescence of island pairs: upon impingement, two islands are held for an interval 
proportional to the fourth power of island radius, and then instantaneously fused into a single hemispherical island with the same total volume.

The average number of islands per cluster of coalescing islands was monitored and found to increase exponentially with time over the period covered by the simulations. When the average number of islands per coalescing cluster traversed 2.0, the film morphology was declared "elongated" with the implication that the percolation transition would occur later by a fixed time factor.

The experimental morphology evolution for various pulse repetition rates is reported in Fig. 4, and the measured percolation transition in the left-hand column of Fig. 5. The simulations reach only the elongation transition, and those results are reported in the right-hand column of Fig. 5. The measurements of the percolation transition, the simulations of the elongation transition, and analytical scaling arguments are all consistent with the transition scaling as pulse frequency to the $-1 / 3$ power at high temperature (corresponding to the "fast substrate diffusion" regime of Jensen and coworkers). At sufficiently low temperature, both experimentally and in the simulations, the scaling behavior shows different power laws over different frequency regimes. We have found some correspondence but we have not found a one-to-one correspondence between the regimes observed in experiment and simulation [49]. It is possible that at sufficiently low temperature, surface faceting and the associated singular surface energetics and kinetics invalidates the classical coalescence kinetics on which the simulation model is based.

These simulations were also used to compare pulsed and continuously deposited films, both with negligible kinetic energy. In this case, the KMC simulations predict that PLD films should 
advance to percolation with less deposition (i.e. they should "percolate faster") than thermally deposited films at the same average deposition rate. This occurs because the higher instantaneous deposition rate in PLD creates a higher density of nucleated small islands, and smaller diameter islands percolate at a smaller average film thickness. At low substrate temperatures, the prediction of faster percolation in PLD is confirmed experimentally, as shown in Fig. 6. However, in situ resistance measurements and ex situ Atomic Force Microscopy topographs demonstrate that at high substrate temperatures, PLD films require more deposition to reach percolation (i.e. they "percolate more slowly")[50]. PLD experiments performed at varying kinetic energy of the depositing Ag species suggest a regime in which increasing kinetic energy can delay the percolation transition. Comparison was made with KMC simulations (Fig. 7) of unconstrained two-island coalescence in the presence of adatom-vacancy pair creation, which occurs with a greater-than-unity yield per incident ion at kinetic energy $>50 \mathrm{eV}$. A surprising mechanism controlling the delayed percolation of PLD films in the high-temperature regime emerged: (1) the energetic deposition results in a net uphill atom flux from adatom-vacancy pair creation, inducing a vertical shape change; (2) taller-than-equilibrium islands coalesce more rapidly; (3) the result is an extended time period over which coalescence is efficient compared to island-island impingement; (4) the percolation transition is delayed.

\section{SUMMARY}

Comparing morphology evolution for PLD and thermal deposition in the same dual-use chamber under identical thermal, background, and surface preparation conditions, other than the 
differing nature of the deposition flux, has been essential to tease out some of the finer distinctions between growth morphology evolution in PLD and thermal deposition.

In semiconductor homoepitaxy, we found that the MBE picture of roughening, mounding, pyramids, and extended defect accumulation to epitaxial breakdown also explains our observations of PLD for kinetic energies up to about $300 \mathrm{eV}$, and that kinetic energy effects promote smoothening and permit epitaxial growth to greater thicknesses without epitaxial breakdown.

In metal-on-insulator film growth, we found that the same morphology progression occurred as in thermal deposition: equiaxed islands, elongated islands, percolating metal film, hole filling. The kinetic freezing model, involving the competition between island-island coalescence and deposition-driven island-island impingement, explains the morphological transitions in both thermal deposition and PLD. KMC simulations based on the kinetic freezing model, with islands constrained to be hemispherical, predict that the rate of progression through the transition is higher with higher pulse repetition rate, which is consistent with the experiments. But in comparing PLD with steady state thermal deposition, the simulations predict a more rapid advancement through the progression in PLD, which is contrary to experiment at high temperature. For low temperatures, the high island density of PLD dominates the morphology evolution, and PLD films reach percolation sooner than thermally deposited films. As the temperature is increased, the PLD percolation thickness approaches and then exceeds the thermal percolation thickness, indicating the increasing importance of an energetic effect. This effect appears to be kinetic energy induced adatom-vacancy pair creation, which has the net effect of moving atoms upward, resulting in a vertical shape transition of the islands. Taller islands 
coalesce more rapidly, thereby delaying the point of the elongation transition, where coalescence is overwhelmed by impingement.

\section{ACKNOWLEDGMENTS}

This research was supported initially by DOE grant DE-FG02-01ER45947 and subsequently by NSF grant DMR-0306997. The results reviewed here are the culmination of the combined efforts of Byungha Shin, Jeff Warrender, John Leonard, Craig Arnold, Jonah Erlebacher and Jim McCamy in my laboratory, and I consider myself fortunate to have worked with them. 


\section{REFERENCES}

${ }^{1}$ J.C. Miller and R.F. Haglund, "Laser Ablation and Desorption," (Academic, 1998).

${ }^{2}$ P.R. Willmott and J.R. Huber, Reviews of Modern Physics 72, 315 (2000).

${ }^{3}$ J. Tersoff, M.D. Johnson, and B.G. Orr, Physical Review Letters 78, 282 (1997).

${ }^{4}$ J.T. Cheung and J.S. Horwitz, MRS Bulletin 17, 30 (1992).

${ }^{5}$ Y. Franghiadakis, C. Fotakis, and P. Tzanetakis, Journal of Applied Physics 84, 1090 (1998).

${ }^{6}$ Y. Franghiadakis, C. Fotakis, and P. Tzanetakis, Appl. Phys. A 68, 391 (1999).

${ }^{7}$ C.B. Arnold and M.J. Aziz, Applied Physics A 69, 23 (1999).

${ }^{8}$ D.B. Chrisey and G.K. Hubler, "Pulsed Laser Deposition of Thin Films," (John Wiley, New York, 1994).

${ }^{9}$ J.S. Horwitz, D.B. Chrisey, R.M. Stroud, A.C. Carter, J. Kim, W. Chang, J.M. Pond, S.W. Kirchoefer, M.S. Osofsky, and D. Koller, Applied Surface Science 129, 507 (1998).

${ }^{10}$ H. Sankur, W.J. Gunning, J. DeNatale, and J.F. Flintoff, Journal of Applied Physics 65, 2475 (1989).

${ }^{11}$ T. Venkatesan, X.D. Wu, R. Muenchausen, and A. Pique, MRS Bull. 17, 54 (1992).

${ }^{12}$ Z.-W. Lai and S. Das Sarma, Physical Review Letters 69, 3762 (1992).

${ }^{13}$ G.H. Gilmer, C. Roland, D. Stock, M. Jaraiz, and T. Diaz de la Rubia, Materials Science and Engineering B-Solid State Materials for Advanced Technology 37, 1 (1996).

${ }^{14}$ J.R. Botha and A.W.R. Leitch, Journal of Crystal Growth 169, 629 (1996).

${ }^{15}$ S. Mackowski, G. Karczewski, F. Kyrychenko, T. Wojtowicz, and J. Kossut, Thin Solid Films 367, 210 (2000).

${ }^{16}$ W. Wulfhekel, H.J.W. Zandvliet, B.J. Hattink, G. Rosenfeld, G. Comsa, and B. Poelsema, Physical Review B 58, 15359 (1998). 
${ }^{17}$ M.I. Larsson and G.V. Hansson, Japanese Journal of Applied Physics Part 1-Regular Papers Short Notes \& Review Papers 33, 2282 (1994).

${ }^{18}$ M.I. Larsson, W.-X. Ni, and G.V. Hansson, J. Appl. Phys. 78, 3792 (1995).

${ }^{19}$ G. Rosenfeld, N.N. Lipkin, W. Wulfhekel, J. Kliewer, K. Morgenstern, B. Poelsema, and G. Comsa, Applied Physics a-Materials Science \& Processing 61, 455 (1995).

${ }^{20}$ G. Rosenfeld, B. Poelsema, and G. Comsa, Journal of Crystal Growth 151, 230 (1995).

${ }^{21}$ G. Koster, G.J.H.M. Rijnders, D.H.A. Blank, and H. Rogalla, Appl. Phys. Lett. 74, 3729 (1999).

${ }^{22}$ M.E. Taylor, H.A. Atwater, and M.V.R. Murty, Thin Solid Films 324, 85 (1998).

${ }^{23}$ H. Jenniches, M. Klaua, H. Hoche, and J. Kirschner, Appl. Phys. Lett. 69, 3339 (1996).

${ }^{24}$ H. Jenniches, J. Shen, C.V. Mohan, S.S. Manoharan, J. Barthel, P. Ohresser, M. Klaua, and J. Kirschner, Physical Review B 59, 1196 (1999).

${ }^{25}$ S.S. Manoharan, J. Shen, H. Jenniches, M. Klaua, and J. Kirschner, Journal of Applied Physics 81, 3768 (1997).

${ }^{26}$ M. Zheng, J. Shen, C.V. Mohan, P. Ohresser, J. Barthel, and J. Kirschner, Appl. Phys. Lett. 74, 425 (1999).

${ }^{27}$ P. Ohresser, J. Shen, J. Barthel, M. Zheng, C.V. Mohan, M. Klaua, and J. Kirschner, Physical Review B 59, 3696 (1999).

${ }^{28}$ M. Weinelt, S. Schwarz, H. Baier, S. Muller, L. Hammer, K. Heinz, and T. Fauster, Physical Review B 63, 205413/1 (2001).

${ }^{29}$ B. Hinnemann, H. Hinrichsen, and D.E. Wolf, Physical Review E 67, 011602 (2003).

${ }^{30}$ M.E. Taylor and H.A. Atwater, Applied Surface Science 127-129, 159 (1998).

${ }^{31}$ B. Shin and M.J. Aziz, Phys. Rev. B 76, 085431 (2007). 
${ }^{32}$ B. Shin, J.P. Leonard, J.W. McCamy, and M.J. Aziz, Appl. Phys. Lett. 87, 181916 (2005).

${ }^{33}$ B. Shin, J.P. Leonard, J.W. McCamy, and M.J. Aziz, J. Vac. Sci. Technol. A 25, 221 (2007).

${ }^{34}$ B. Shin and M.J. Aziz, Phys. Rev. B 76, 165408 (2007).

${ }^{35}$ P. Politi and J. Villain, Physical Review B 54, 5114 (1996).

${ }^{36}$ K.A. Bratland, Y.L. Foo, J. Soares, T. Spila, P. Desjardins, and J.E. Greene, Physical Review B 67, 125322 (2003).

${ }^{37}$ D.L. Smith, C.C. Chen, G.B. Anderson, and S.B. Hagstrom, Appl. Phys. Lett. 62, 570 (1993).

${ }^{38}$ E. Vasco and J.L. Sacedon, Physical Review Letters 98, 036104 (2007).

${ }^{39}$ D. Walton, J. Chem. Phys. 37, 2182 (1962).

${ }^{40}$ J.S. Biteen, D. Pacifici, N.S. Lewis, and H.A. Atwater, Nano Letters 5, 1768 (2005).

${ }^{41}$ J.S. Biteen, N.S. Lewis, H.A. Atwater, H. Mertens, and A. Polman, Appl. Phys. Lett. 88, 131109 (2006).

${ }^{42}$ G. Jeffers, M.A. Dubson, and P.M. Duxbury, Journal of Applied Physics 75, 5016 (1994).

${ }^{43}$ J. Carrey and J.-L. Maurice, Phys. Rev. B 63, 245408 (2001).

${ }^{44}$ J. Carrey and J.-L. Maurice, Phys. Rev. B 65, 205401 (2002).

${ }^{45}$ F.A. Nichols and W.W. Mullins, Journal of Applied Physics 36, 1826 (1965).

${ }^{46}$ A.A. Baski and H. Fuchs, Surface Science 313, 275 (1994).

${ }^{47}$ P. Jensen and B. Niemeyer, Surface Science 384, L823 (1997).

${ }^{48}$ N. Combe and P. Jensen, Phys. Rev. B 57, 15553 (1998).

${ }^{49}$ J.M. Warrender and M.J. Aziz, Phys. Rev. B 76, 045414 (2007).

${ }^{50}$ J.M. Warrender and M.J. Aziz, Phys. Rev. B 75, 085433 (2007).

${ }^{51}$ J.M. Warrender and M.J. Aziz, Applied Physics A 79, 713 (2004). 


\section{FIGURES}
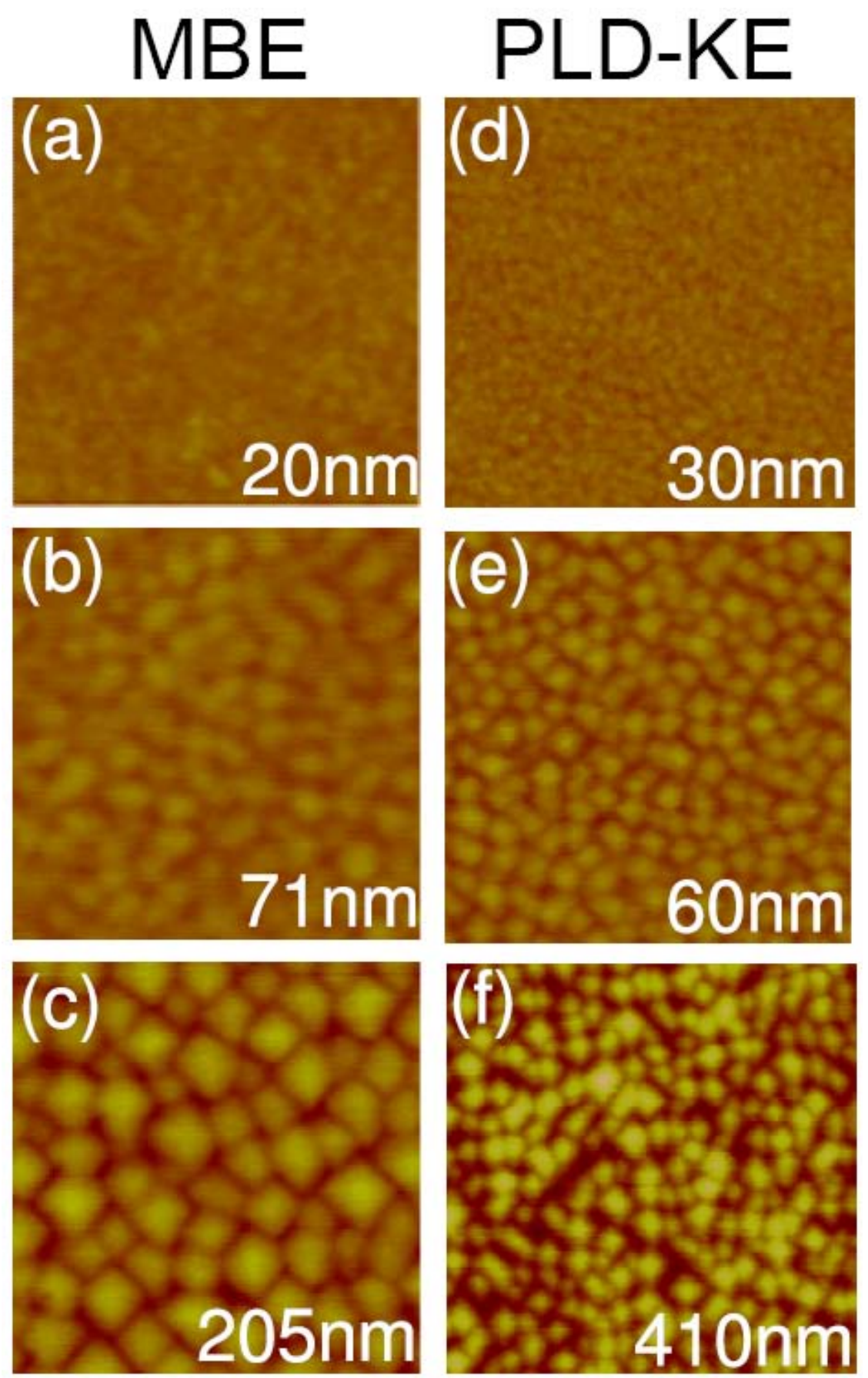

Fig. 1. AFM images of growth morphologies in Ge homoepitaxy. Left column: MBE; right column PLD with peak ion kinetic energy $300 \mathrm{eV}$. Both cases are characterized by the development of roughness (a) and (d)), growth mounds (b) and (e), and pyramidal shapes (c) and (f), before epitaxial breakdown and transition to amorphous phase (not shown). Scan edge length is $0.5 \mu \mathrm{m}$; vertical scale is $10 \mathrm{~nm}$. Film thickness is shown in the right bottom corner of each image. Adapted from ref.[32]. 


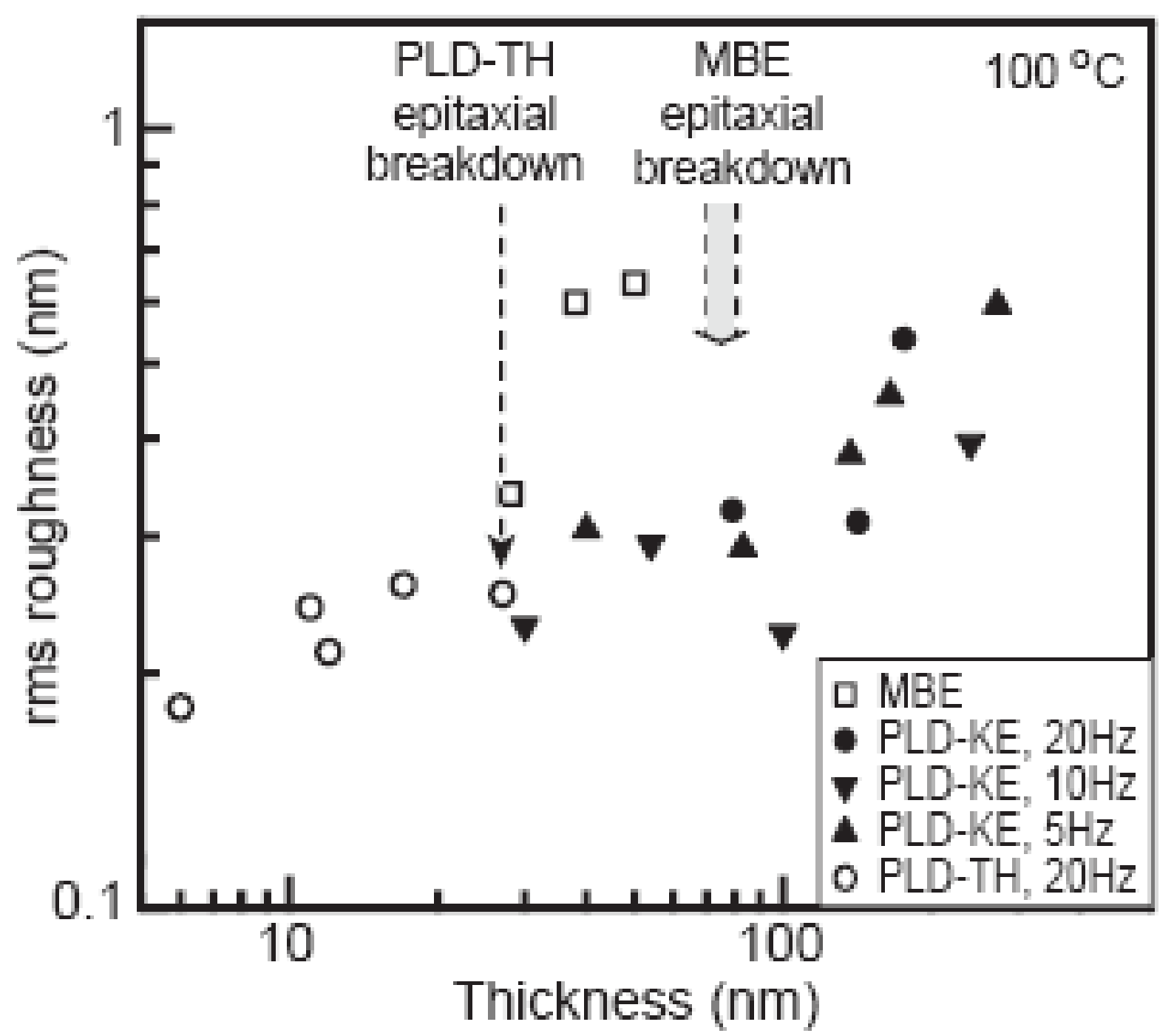

Fig. 2. RMS roughness vs. Ge film thickness and thickness of epitaxial breakdown in Ge homoepitaxy by MBE, PLD with peak ion kinetic energy 300 eV (PLD-KE), and PLD with thermal kinetic energy (PLD-TH). From ref. [31]. 


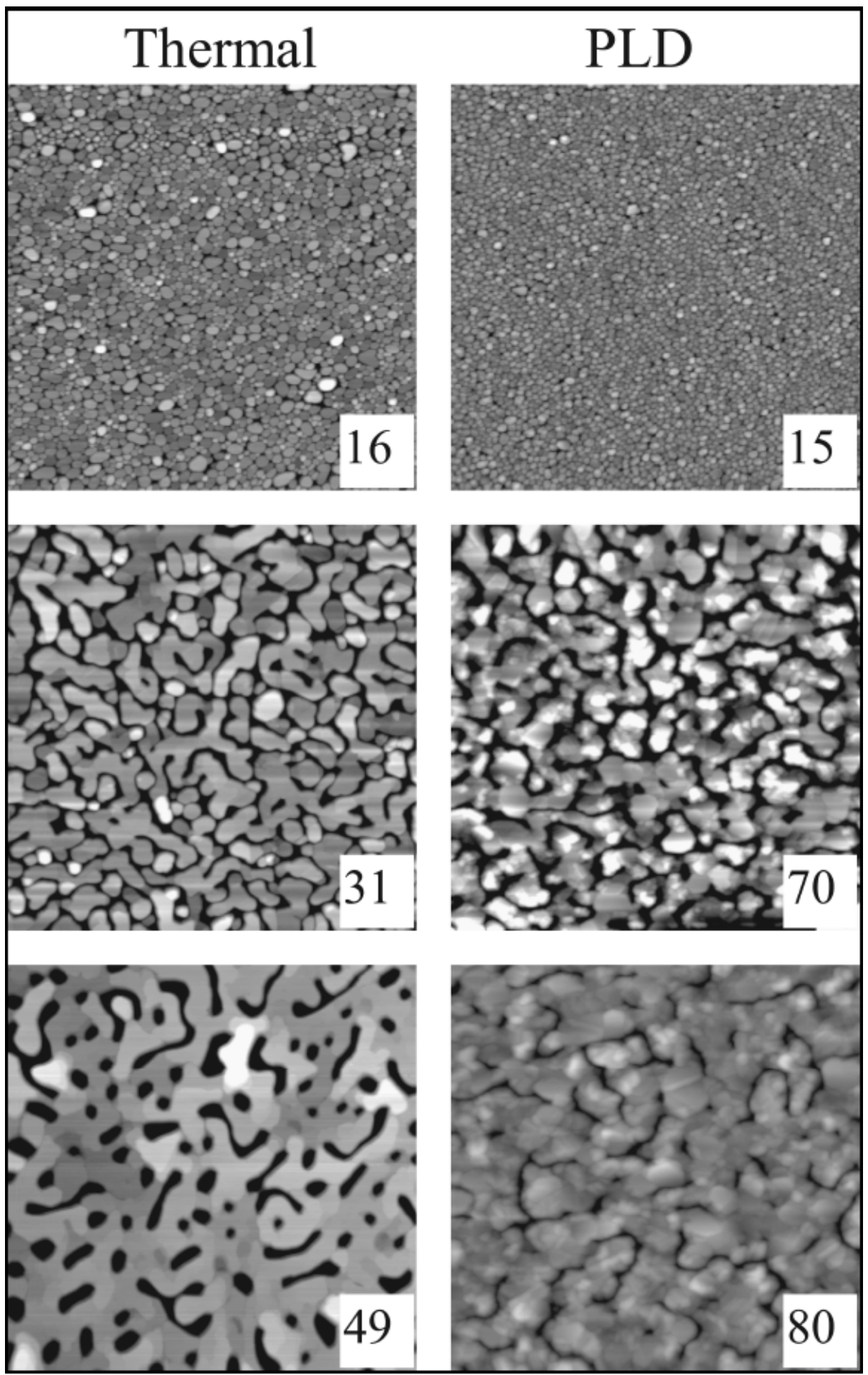

Fig. 3. Metal-on-insulator Volmer-Weber growth mode, illustrating transition from equiaxed islands (top row) to extended, non-percolating islands (middle row) to a percolating metal film with holes filling in (bottom row). Ag on mica; scan edge length is $5 \mu \mathrm{m}$. Inset is average film thickness. Adapted from ref.[49]. 
a Islands Channels Holes
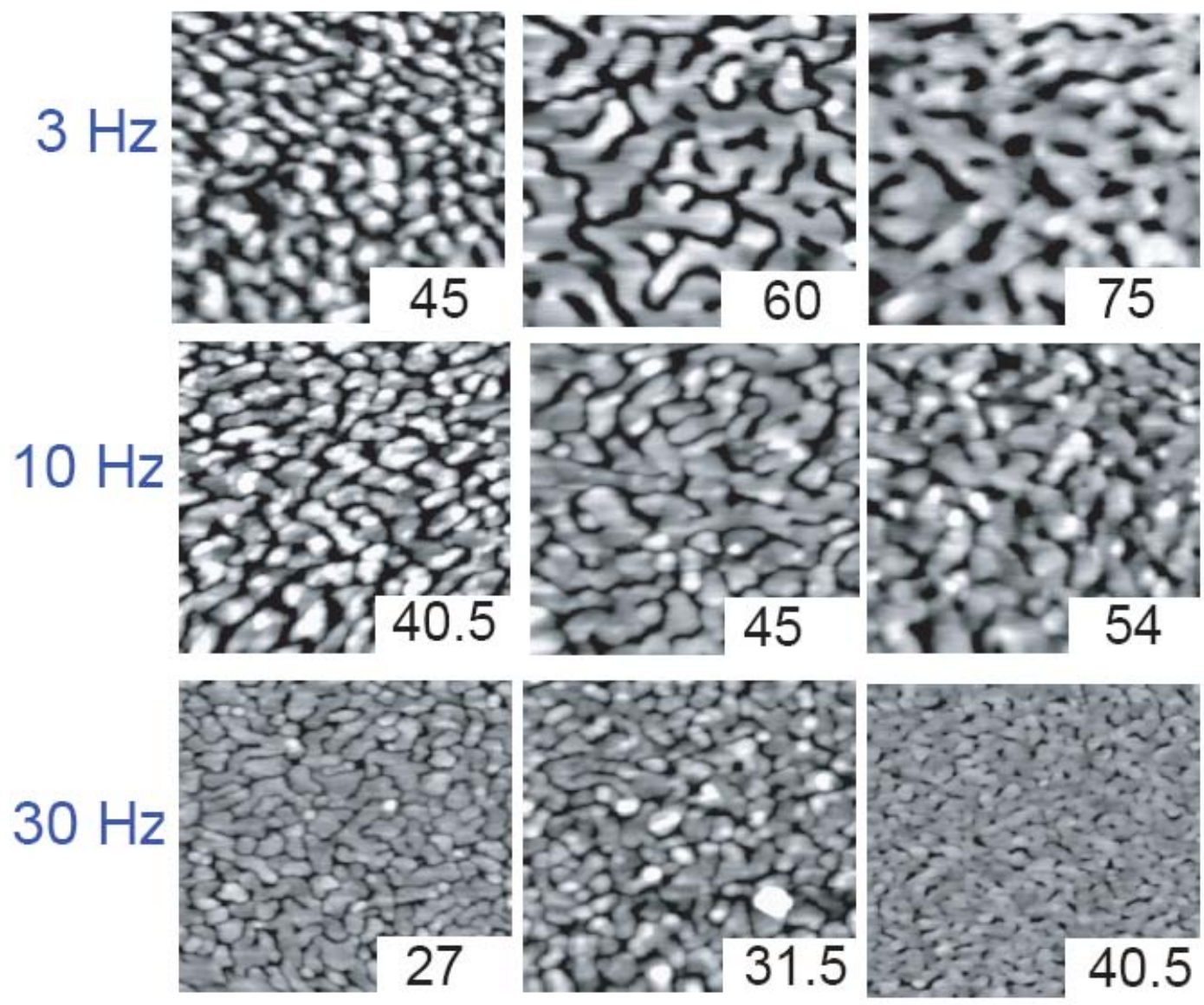

b
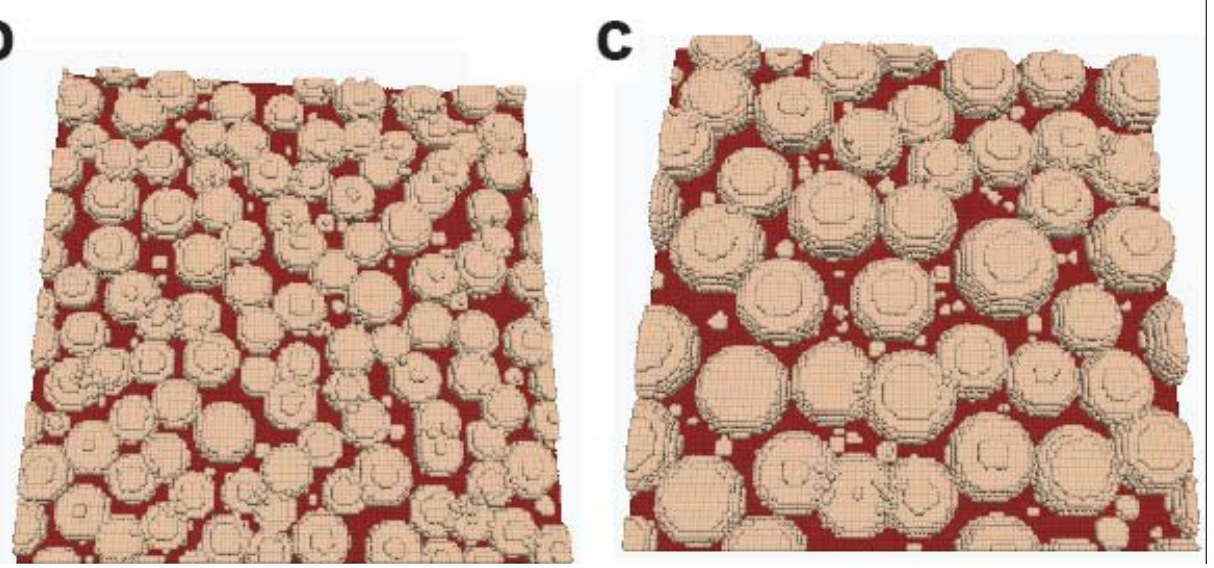

Fig. 4. (a) AFM images of Ag/mica morphology evolution for three different laser pulse repetition rates at constant laser fluence. Film thickness in $\mathrm{nm}$ is indicated in corner of each image. Films deposited at higher pulse rate advance through the progression with lower transition thickness. Scan edge length is $3 \mu \mathrm{m}$. Bottom: Simulated film morphologies at 0.025 $\mathrm{ML} /$ pulse; snapshots with hemispherical islands just reaching the elongation transition: (b) 100 pulses, $100 \mathrm{~Hz}$; (c) 176 pulses, $10 \mathrm{~Hz}$. Smaller islands reach elongation transition at lower average film thickness. Adapted from ref. [51]. 

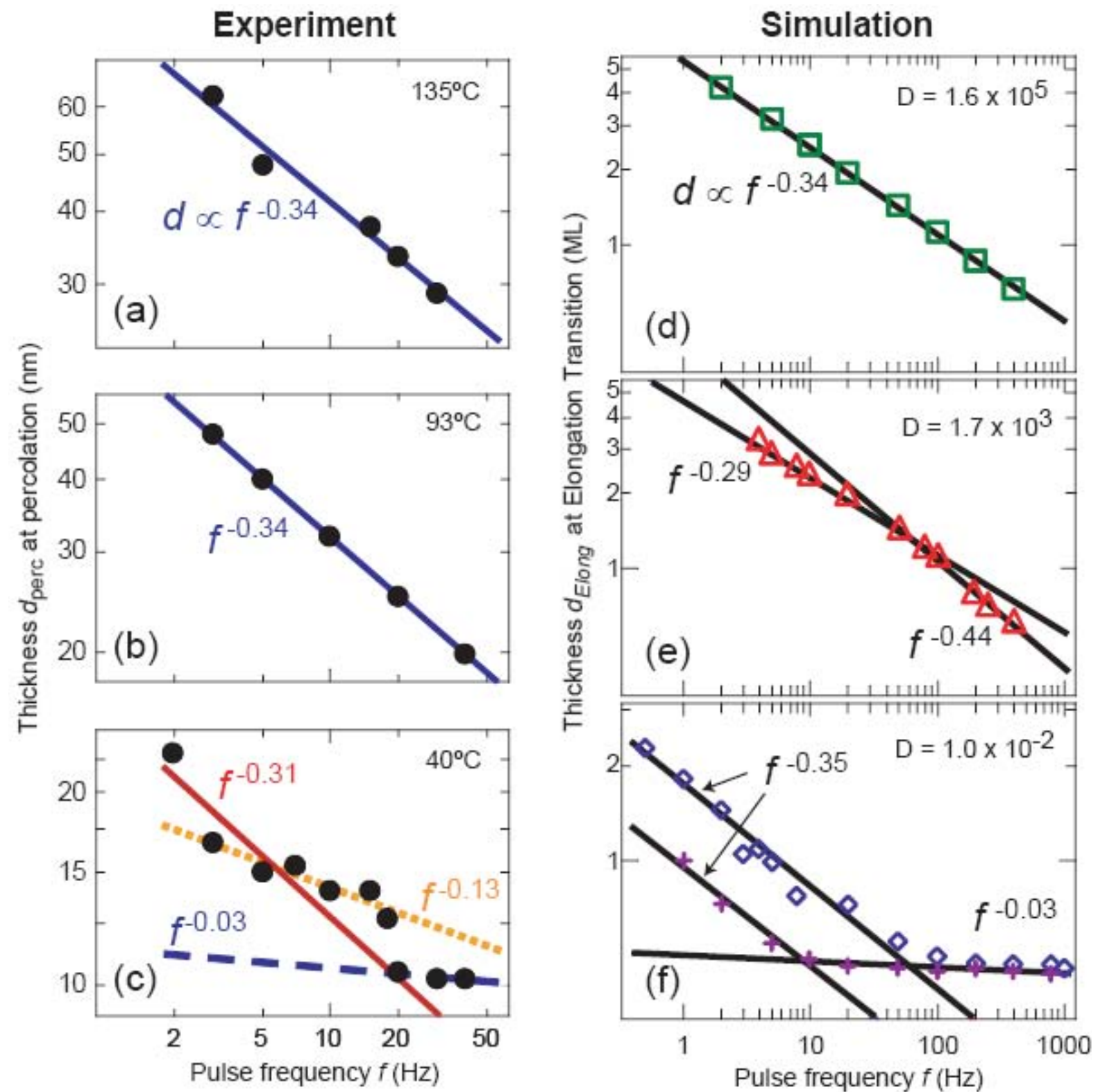

Fig. 5. Film thickness at morphology transition vs. pulse frequency for constant amount deposited per pulse. Top row: high temperature; middle row: intermediate temperature; bottom row: low temperature. From ref. [49]. Experiments measure percolation transition whereas simulations track elongation transition. Simulations in (f) show same power laws for two different values of coalescence rate constant. 

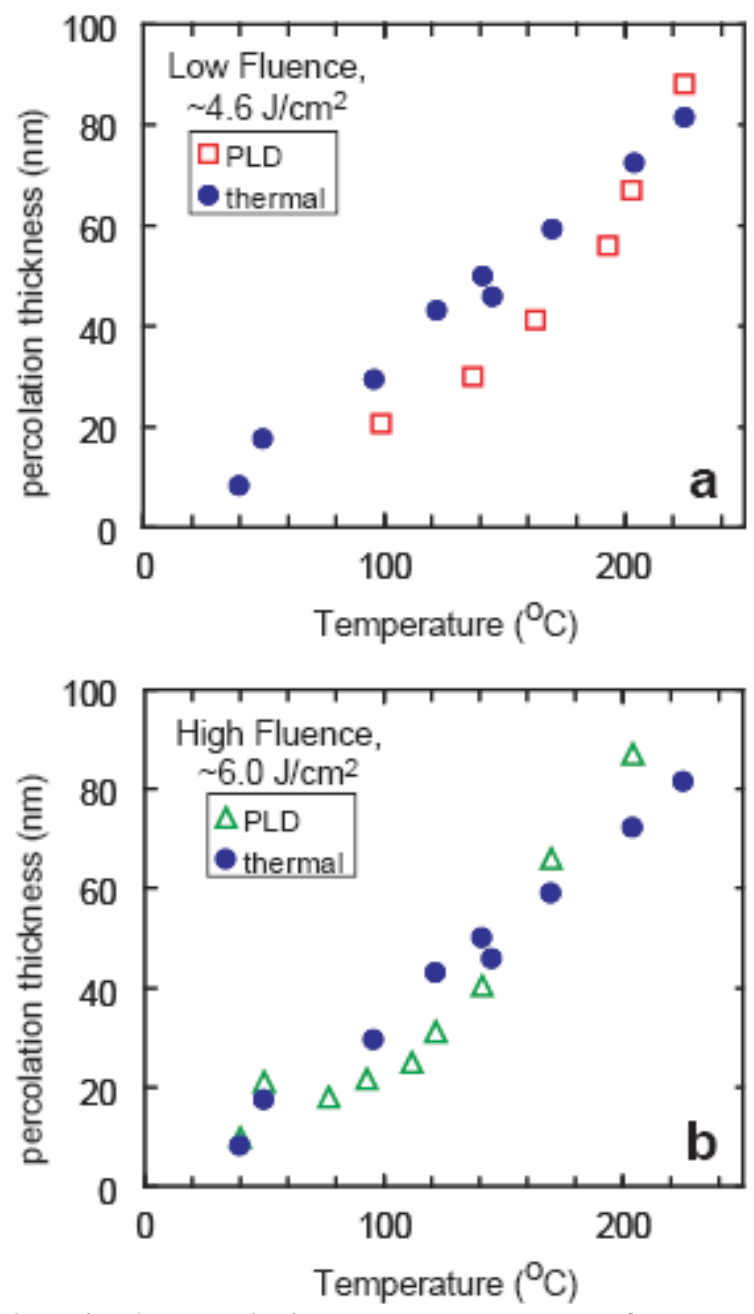

Fig. 6. Film thickness at electrical percolation vs. temperature for PLD (open symbols) and thermal deposition (filled circles; same data shown in both panels for comparison). Average deposition flux was $0.06 \mathrm{~nm} / \mathrm{s}$. Peak ion kinetic energy in PLD was $55 \mathrm{eV}$ in top panel and 110 $\mathrm{eV}$ in bottom panel. Adapted from ref. [50]. 


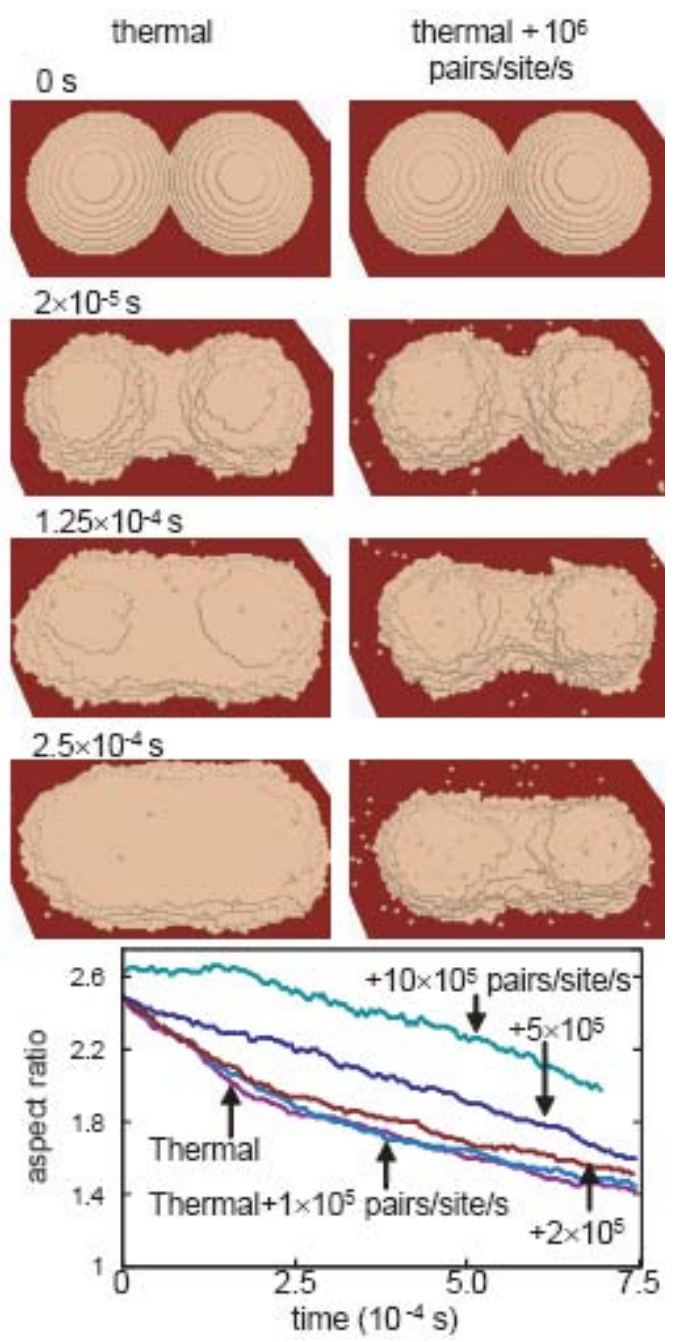

Fig. 7. KMC simulation of evolution with time of coalescing two-island system in absence of deposition. Left column: purely thermal processes; right column superposes energetic mechanism of adatom/vacancy pair creation. Energetic mechanism induces islands to remain taller, and taller islands coalesce more slowly. Plot shows change in aspect ratio of island pair vs. time as two islands equilibrate with several different adatom-vacancy pair creating rates. From ref. [50]. 